\title{
HIGH BITRATE OPERATION OF A NOVEL OPTICAL PHASE CONJUGATOR USING INLINE FIBRE DFB LASERS
}

\author{
S.Y. Set, S. Yamashita, M. Ibsen, R.I. Laming \\ Optoelectronics Research Centre, University of Southampton, Southampton SO17 1BJ, UK \\ Tel: +44 1703 593138, Fax: +44 1703 593142, (email: sys@orc.soton.ac.uk)
}

D. Nesset, A.E. Kelly and C. Gilbertas

BT Laboratories, Martlesham Heath, Ipswich, Suffolk IP5 3RE, UK

\begin{abstract}
We report the first demonstration of high bitrate, polarisation insensitive optical phase conjugation and wavelength conversion using orthogonally polarised fibre distributed feedback lasers as four-wave mixing pumps in both dispersion shifted fibre and semiconductor optical amplifier.
\end{abstract}

Dispersion shifted fibre (DSF) and semiconductor optical amplifier (SOA) are two of the most promising media for optical phase conjugation and wavelength conversion using FWM [1,2]. These are key functions in future high bitrate transmission systems for dispersion compensation using MSSI $[3,4]$ and switching in WDM networks. Key issues in FWM are the efficiency and polarisation independence. The efficiency has been increased by using both novel fibres [5] and SOAs [6]. Polarisation independent FWM has been demonstrated in both SOA [7] and in DSF [8], using two orthogonally polarised pumps. With this technique, $40 \mathrm{Gbit} / \mathrm{s} 102 \mathrm{~km}$ MSSI transmission has been demonstrated [9]. However this configuration results in high complexity and component count.

In this paper, we report the use of a novel optical phase conjugator configuration [10] in 20/40Gbit/s system experiments. The conjugator uses inline fibre distributed feedback (DFB) lasers as pumps and polarisation independent operation is achieved by aligning the polarisations orthogonally. This simple configuration eliminates the need for the additional amplifiers and couplers required by the conventional dual-pumped setup [10]. Here, we apply this novel device to FWM in both a highly nonlinear DSF (HNL-DSF) [5] and a long SOA [6] to achieve wideband and high conversion efficiency.

Fig. 1 shows the experimental setup. The signal source is a $10 \mathrm{GHz}$, transform limited 5 ps pulse train generated using a tandem electroabsorption modulator (EAM) configuration [9]. It is externally modulated using a lithium niobate $\left(\mathrm{LiNbO}_{3}\right)$ modulator driven by a $10 \mathrm{Gbit} / \mathrm{s}$, $2^{31}-1$ pseudo-random bit sequence (PRBS) and interleaved by a 10 to $20 / 40 \mathrm{Gbit} / \mathrm{s}$ interleaver. The interleaver is made from PM fibre and all channels are co-polarised, and delayed with respect to each other by $\sim 10 \mathrm{~ns}$ to decorrelate the data patterns. We launch the 20/40Gbit/s signal directly into two inline cascaded, single polarisation $\mathrm{Er}^{3+}: \mathrm{Yb}^{3+}$ fibre DFB lasers which serve as the FWM pumps at $1551.5 \mathrm{~nm}$ and $1552.8 \mathrm{~nm}$. Each of the fibre DFB lasers is optically isolated for stability and pumped by separate $110 \mathrm{~mW}, 980 \mathrm{~nm}$ diodes to give flexibility in power balance of the pumps. The $1.3 \mathrm{~nm}$ wavelength detuning of the pumps is near the minimum required for high bitrate operation [8] as in the SOA case, polarisation dependence increases with this detuning [9]. A $23 \mathrm{dBm}$ high power optical amplifier is used to boost the signal and pumps before they are launched into the nonlinear mixing media, which is either $440 \mathrm{~m}$ of HNL-DSF or a 2 $\mathrm{mm}$ long SOA at high injection current $550 \mathrm{~mA}$.

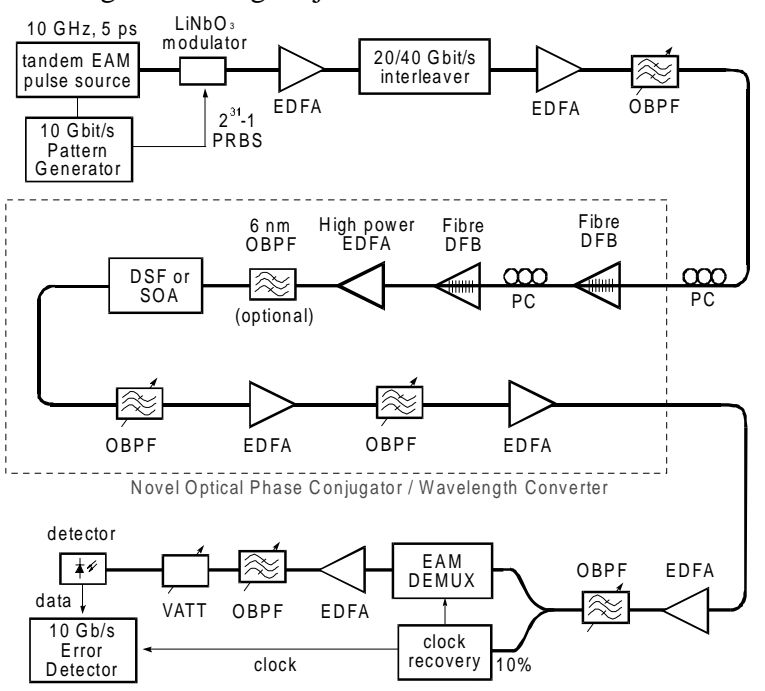

Figure 1: Experimental setup

The phase conjugate signal generated by FWM is subsequently filtered and amplified by two $1.3 \mathrm{~nm}$ polarisation insensitive optical bandpass filters (OBPFs) and two erbium-doped fibre amplifiers (EDFAs). At the receiver end, $10 \%$ of the conjugated signal is coupled out for clock recovery. The derived electrical clock drives the EAM (DEMUX) and the $10 \mathrm{Gbit} / \mathrm{s}$ demultiplexed channel is fed to a receiver and error detector.

Fig. 2(a) and (b) show the output spectra of the DSF- and SOA-based conjugators respectively. In the SOA case, the signal wavelength is tuned closer to the pumps for higher conversion efficiency and a $6 \mathrm{~nm}$ OBPF is inserted to filter the out-of-band ASE. The state of polarisations (SOPs) of the two fibre DFB lasers are aligned using a polarisation controller (PC) inserted between them. Orthogonal SOPs could be achieved by monitoring the output spectrum of the conjugator and minimise the pump1-pump2 FWM components, PP1 and PP2 (Fig. 2), to $40 \mathrm{~dB}$ below the 
pumps. Spectrally, it can be seen that any changes in signal SOP do not change the conjugate (C3) level significantly, hence polarisation independent operation is demonstrated.
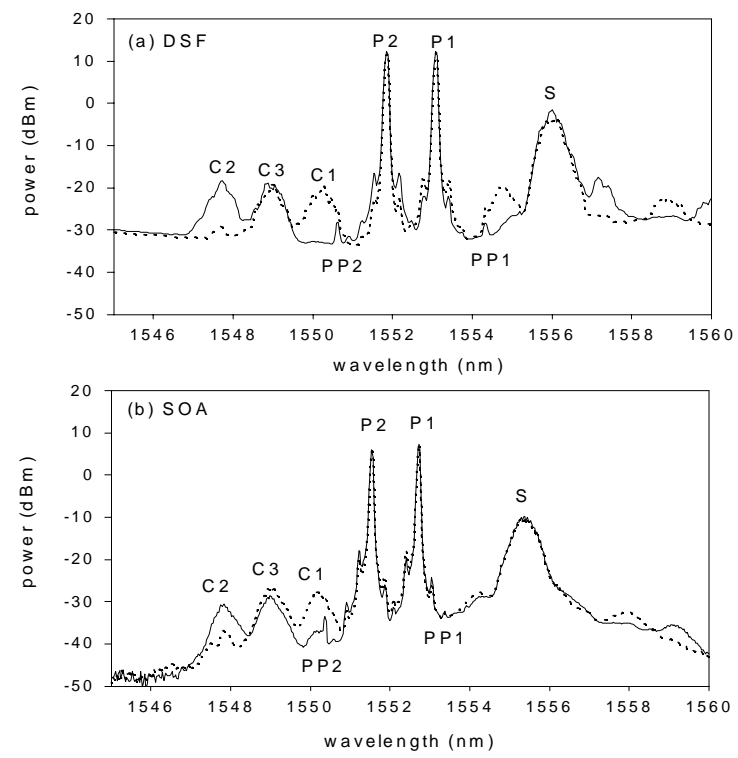

Figure 2: Optical spectra of (a) DSF- and (b) SOAbased conjugators (solid lines and dotted lines represent two extreme signal polarisation cases)

The BER results of $20 \mathrm{Gbit} / \mathrm{s}$ optical phase conjugation are shown in Fig.3(a). Polarisation sensitivity as low as 0.5 $\mathrm{dB}$ in power penalty is achieved in the DSF-based conjugator. In an SOA, polarisation induced power penalty is measured to be $1.5 \mathrm{~dB}$ due to extra polarisation dependence in the $6 \mathrm{~nm}$ OBPF. We have error free results at a received power of $>-10 \mathrm{dBm}$ despite signal polarisation fluctuations.
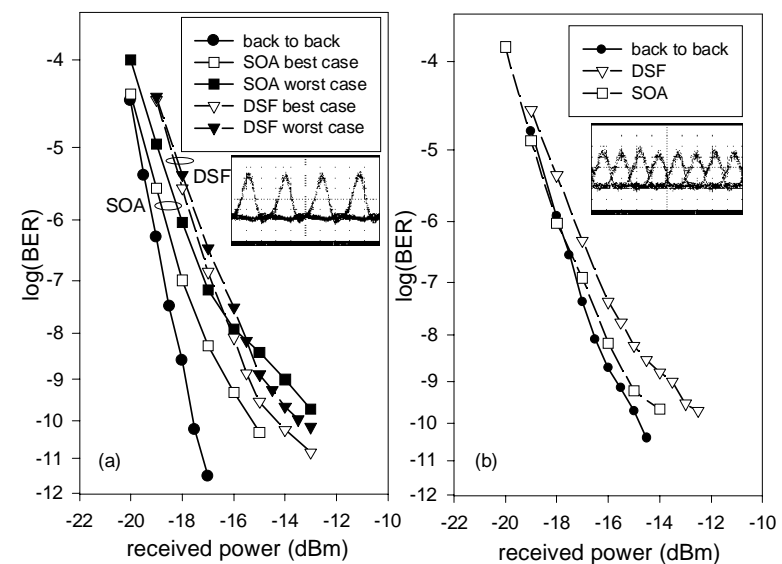

Figure 3: Bit error rate performance: (a) $20 \mathrm{Gbit} / \mathrm{s}$, polarisation independent and (b) $40 \mathrm{Gbit} / \mathrm{s}$

Fig 3(b) shows $40 \mathrm{Gbit} / \mathrm{s}$ operation with a single fibre DFB laser pump (polarisation sensitive). However, in the polarisation insensitive configuration, we have encountered error floor at around $10^{-8 \sim} 10^{-9}$ error rate. This is due to the ASE-noise at the conjugate wavelength affecting conjugate signal-to-noise ratio. Despite the error floor, we have carried out MSSI transmission over $204 \mathrm{~km}$ of standard fibre at $40 \mathrm{Gbit} / \mathrm{s}$. The received demultiplexed signal shows good eyes with low polarisation dependency in the SOA-based conjugator (Fig.4) and similar performance is obtained DSF.

In order to improve the error performance, one could increase the power of the DFB pumps thus eliminating the need for the amplifier prior to the nonlinear media. This could be done by using a dual-pumped fibre DFB for example.

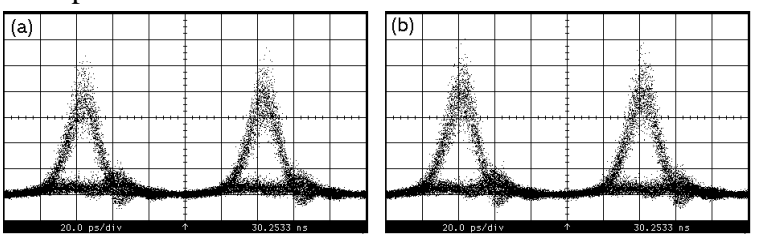

Figure 4: Eye diagrams of $204 \mathrm{~km}$ MSSI transmission: (a) minimum and (b) maximum received signal

In summary, we have demonstrated high bitrate optical phase conjugation and wavelength conversion with a novel fibre DFB laser pumped configuration. This configuration has shown good performance both with SOA and DSF at $20 \mathrm{Gbit} / \mathrm{s}$. With the use of a HNL-DSF or a long SOA, practical polarisation insensitive, wideband phase conjugators are realisable, suitable for future WDM and MSSI applications.

The authors would like to thank Dr M. Onishi and Sumitomo Electric Industries, Ltd. for the provision of the highly nonlinear DSF. This work is partially supported by the European Commission through the ACTS projects 'MIDAS' and 'HIGHWAY'. The ORC is an EPSRC funded interdisciplinary research centre.

\section{References}

[1] K. Inoue and H. Toba, IEEE Photon. Technol. Lett., vol. 4, pp. 69-72, 1992.

[2] M.C. Tatham, G. Sherlock, and L.D. Westbrook, IEEE Photon. Technol. Lett., vol. 5, pp. 1303-1306, 1993.

[3] S. Watanabe, T. Naito, and T. Chikama, IEEE Photon. Technol. Lett., vol. 5, pp. 92-95, 1993.

[4] A. Royset, S.Y. Set, I.A. Goncharenko, and R.I. Laming, IEEE Photon. Technol. Lett., vol. 8, no. 3, pp. 449-451, 1996.

[5] M. Onishi, T. Okuno, T. Kashiwada, S. Ishikawa, N. Akasaka, and M. Nishimura, Proc. ECOC'97, vol. 2, pp. 115-118, 1997.

[6] A.E. Kelly, D.D. Marcenac, and D. Nesset, Electron. Lett., vol. 33, no. 25, 1997.

[7] R.M. Jopson and R.E. Tench, Electron. Lett., vol. 29, no. 25, pp.2216-2217, 1993.

[8] K. Inoue, J. Lightwave Technol., vol. 12, no. 11, pp. 1916-1920, 1994.

[9] D.D. Marcenac, D. Nesset, A.E. Kelly and D. Gavrilovic, Electron. Lett., vol. 33, no. 1, pp. 100101, 1998.

[10]S. Yamashita, S.Y. Set, and R.I. Laming, "A novel polarisation independent phase conjugator/ wavelength converter utilising inline fibre DFB lasers," submitted to Electron. Lett., 1998. 